ArtefaCToS. Revista de estudios de la ciencia y la tecnología

eISSN: 1989-3612

Vol. 7, No. 1 (2018), 2a Época, 143-153

DOI: http://dx.doi.org/10.14201/art201871143153

\title{
Objetividad y racionalidad en la economía del conoci- miento científico
}

\section{Objectivity and Rationality in Scientific Knowledge Economy}

\author{
Jesús ZAMORA BONILLA
}

Universidad Nacional de Educación a Distancia

jpzb@fsof.uned.es

Recibido: 16/01/2018. Revisado: 20/01/2018. Aceptado: 24/01/2018

\section{Resumen}

Se presentan las líneas generales de una "economía del conocimiento científico", en particular, de un enfoque de los estudios sociales de la ciencia basado en la aplicación de la teoría de juegos al análisis de las interacciones e instituciones científicas. Se plantea la cuestión de en qué sentido y en qué medida un enfoque de este tipo puede ser útil para responder a las clásicas preguntas sobre la racionalidad y la objetividad del conocimiento científico. Estas cuestiones se ilustran con la aplicación de la teoría de juegos a tres situaciones diferentes: la elección de normas o estándares de calidad en la ciencia, la elección de una teoría, y la "construcción social" de un hecho científico.

Palabras clave: racionalidad; objetividad; economía del conocimiento científico; estudios sociales de la ciencia; valor epistémico; teoría de juegos; modelos.

\begin{abstract}
The paper presents the general lines of an "economy of scientific knowledge", and specifically an approach from the social studies of science based on the application of game theory to the analysis of scientific interactions and institutions. The paper will analyze in what sense and to what extent an approach of this kind can be useful to answer the classic questions about the rationality and objectivity of scientific knowledge. These issues are exemplified with the
\end{abstract}

(C) Ediciones Universidad de Salamanca - CC BY-NC-ND 
application of game theory to three different situations: the choice of standards or quality standards in science, the choice of a theory, and the "social construction" of a scientific fact.

Keywords: Rationality; Objectivity; Economics of Scientific Knowledge; Social Studies of Science; Epistemic Value; Game Theory; Models.

\section{Introducción}

A lo largo de las últimas dos décadas buena parte de mi investigación la he dedicado al desarrollo de lo que podemos llamar un enfoque "económico" para la comprensión de la "construcción social del conocimiento científico", basado sobre todo en la aplicación de la teoría de la elección racional y la teoría de juegos (ver Fernández Pinto, 2016; Zamora Bonilla 2011). Un enfoque así complementaría los esfuerzos tradicionales en esta área, que utilizan ideas y técnicas de otras disciplinas de las ciencias sociales, tales como la sociología y la antropología, pero que aportaría también dos virtudes fundamentales de una perspectiva "racionalista": en primer lugar, el enfoque de elección racional permite modelizar de manera explícita los factores que determinan las decisiones de los científicos, así como las interdependencias entre estos factores, sin la necesidad de revestir a estos factores de una retórica mistificadora, que más bien contribuye a oscurecer el análisis más que a iluminarlo. En segundo lugar, en vez de una acusación genérica de falta de objetividad y racionalidad a los productos y a los métodos de la investigación científica, como hacen algunos enfoques sociologistas tendentes al relativismo, los modelos económicos nos permiten especificar claramente cuáles pueden ser las deficiencias específicas que pueden conllevar algunas formas de llevar a cabo los procesos de investigación, es decir, nos permiten detectar modos concretos de ineficiencia, e iluminarnos en la búsqueda de maneras mediante las cuales resolverlas o mejorarlas. Dicho de otro modo, un análisis de la construcción social del conocimiento científico en términos de teoría de juegos nos permite no renunciar a la tesis de que la ciencia es un método relativamente eficaz en proporcionarnos verdades objetivas e interesantes sobre el mundo, ni a la tesis de que la ciencia es el producto de fuerzas típicamente sociales y humanas. Y, sobre todo, nos permite no renunciar al objetivo de encontrar maneras en las que mejorar el modo en que la ciencia persigue aquellos fines. En particular, voy a dedicar este artículo a intentar explicar mediante algunos ejemplos de qué forma los modelos de teoría de juegos pueden contribuir a entender la objetividad y la racionalidad de la ciencia. 


\section{Los elementos de un modelo de teoría de juegos sobre la ciencia}

Para entender la construcción del conocimiento científico mediante el uso de la teoría de juegos necesitamos adoptar un enfoque cognitivo que no es frecuente en los llamados "science studies" (aunque sí es más común en otros enfoques de filosofía de la ciencia, como por ejemplo el Bayesianismo). Me refiero, por supuesto, al diseńo de modelos abstractos. Un modelo formal puede ser considerado como un argumento esquematizado, con el que se intenta probar que ciertas conclusiones interesantes se siguen (o que no se siguen) a partir de ciertas premisas razonables. Este estilo argumentativo contrasta fuertemente con el que es más habitual en los estudios sociales de la ciencia: el "estudio de casos". Por supuesto, no hay nada que objetar a ese tipo de estudios basados en la descripción "sociológica" o "histórica" de casos concretos, es más, se trata de una actividad absolutamente necesaria para conocer y entender la producción del conocimiento científico, pero la "tecnología de estudios de casos" es esencialmente inductiva, y no muy adecuada, por tanto, para iluminar los mecanismos regulares que hacen que la ciencia sea como es, y por tanto, las conclusiones que alcancemos con ella podrán difícilmente ser generalizados, como muestra el hecho de que conclusiones filosóficas o epistemológicas totalmente distintas hayan podido ser justificadas todas ellas mediante los adecuados "estudios de casos" preferidos por sus autores. De todas formas, no pretendo considerar el enfoque de teoría de juegos como una alternativa a los estudios de casos, sino más bien como un complemento: la idea es tomar el conjunto de esos estudios descriptivos como un corpus de hechos empiricos sobre la ciencia, meramente descriptivos, y utilizar la teoría de la elección racional como una herramienta para intentar descubrir mecanismos teóricos (o sea, modelos abstractos) que nos permitan explicar (al menos parcialmente) por qué la ciencia es como aquellos hechos empíricos dicen que es.

Los principios básicos de un enfoque como el que sugiero son los siguientes (para más detalles, ver Zamora Bonilla 2006a): En primer lugar, el enfoque señala a las acciones o decisiones de los científicos como el elemento básico de lo que tiene que ser explicado. O sea, se trata de entender por qué los científicos hacen lo que hacen y de la forma en que lo hacen. Esto no implica que otros aspectos de los "constructos" científicos (p.ej., la estructura de las teorías, la relación entre modelos y observaciones, etc.) caigan fuera de nuestro enfoque, pues podemos preguntarnos por qué los científicos eligen teorías con cierta estructura, por qué prefieren modelos con tales o cuales tipos de conexiones con los datos, etc. En segundo lugar, un enfoque de elección racional nos obliga a considerar explícitamente los objetivos o fines de que los científicos están persiguiendo mediante sus acciones y decisiones, así como la información, las posibilidades alternativas, y los mecanismos sociales que les permiten alcanzar aquellos fines en la medida en que los consiguen alcanzar. Por último, en tercer lugar, lo que el enfoque de teoría de juegos añade como su aspecto más característico es la idea de que, en la medida en que las decisiones de los otros colegas (u otros agentes relevantes) puedan influir en los resultados que obtiene un científico por su participación en esa actividad, 
las situaciones sociales que podamos esperar encontrar en la realidad tendrán que ser lo que se denomina un "equilibrio de Nash", es decir, una situación en la que cada agente esté eligiendo la mejor alternativa que puede como respuesta a lo que están eligiendo los demás.

Con el objeto de ilustrar estos principios en su aplicación a algunos problemas tradicionales de la filosofía de la ciencia, voy a ocuparme en el resto del artículo de los siguientes ejemplos:

Estándares científicos (Ferreira y Zamora Bonilla, 2006; Zamora Bonilla, 2002): muchos filósofos han discutido a lo largo del tiempo las virtudes que un enunciado científico debe tener para ser "aceptable" (p.ej., grado de confirmación, corroboración, verosimilitud, simplicidad, capacidad predictiva, etc.), pero, puesto que estas virtudes son graduales, podemos razonablemente preguntar cómo determinan de hecho los científicos el grado mínimo de ellas que un enunciado debe superar para que su aceptación en la comunidad científica relevante sea "obligatoria". Dicho de otra manera, ¿bajo qué circunstancias se verá obligado un científico a reconocer que la teoría propuesta por un "rival" es la correcta? Un análisis en términos de teoría de juegos de esta situación muestra que cada elección colectiva de un estándar o de otro más alto o más bajo lleva asociadas unas probabilidades distintas de "ganar" la "carrera por un descubrimiento" (p.ej., si el grado es demasiado elevado, hay más probabilidad de que ningún científico la gane), y ello puede dar a los investigadores una razón suficiente para preferir unos estándares en vez de otros.

Elección de teoría (Zamora Bonilla 1999; 2007): No todos los enunciados científicos en un determinado campo son "obligatorios", en el sentido de que, si un científico decide no aceptar alguno de ellos, no por esa razón va a ser expulsado de la comunidad, o a no reconocérsele como un profesional debidamente cualificado; dicho de otra manera, sobre algunas cuestiones la comunidad puede no haber alcanzado una respuesta consensuada. La famosa tesis filosófica de la "infradeterminación de las teorías por la observación" es simplemente una justificación formal de este hecho, aunque su nombre habitual lleva a confusión, porque no solo se aplica a "teorías", sino en realidad a cualquier enunciado científico, desde los informes de experimentos a los "megaparadigmas". El problema es que decir que "la lógica no es suficiente para determinar la elección de teorías" no nos ayuda mucho a saber de qué factores si que depende en concreto esa elección: ¿son esos factores "intereses sociales", "sesgos cognitivos", o meramente una cuestión de "psicología de masas"? El enfoque de teoría de juegos nos permite decir que, en la medida en que las ventajas que para un científico tenga aceptar un enunciado dependan de cuáles y cuántos de sus colegas lo estén aceptando, entonces las únicas situaciones estables serán aquellas que constituyan un equilibrio, en el sentido indicado más arriba. En general, sólo habrá unos pocos equilibrios en cada caso (aunque a menudo más de uno), y es posible que se den cambios súbitos de un equilibrio a otro (algo así como "revoluciones científicas"), 
pero también es posible probar que, en la medida en que cómo de ventajoso sea aceptar un enunciado dependa también parcialmente de cómo de ese enunciado sea según los estándares epistémicos aceptados por la comunidad, podemos esperar que los enunciados "mejores" tiendan a ser más "populares" entre los científicos que los "peores", a medida que ese grado de calidad epistémica aumenta.

La construcción de un hecho empirico (Zamora Bonilla, 2006b): Hoy en día es una perogrullada dentro del campo de los "science studies" afirmar que la manera en que los "descubrimientos" empíricos son presentados es el resultado de una "negociación". Esto implica, cuando menos, que cada hallazgo empírico puede ser presentado de más de un modo. Pero no implica de ninguna manera que todos esos modos sean igual de buenos para cada agente involucrado en la "negociación". Antes, al contrario, si todos ellos fueran "igual de buenos", no habría negociación en absoluto (pues la negociación tiene un coste), y la cuestión podría resolverse pacíficamente mediante un sorteo, por ejemplo. La modelización mediante juegos de este tipo de situaciones nos permite ver que los intereses y preferencias de distintos "negociadores" puede inducir una distinción entre aquellas interpretaciones de un descubrimiento que pueden ser el resultado de una negociación, y aquellas que directamente no pueden. También muestra algunas formas en las que el resultado de la negociación puede ser considerado más o menos eficiente, y formular instituciones que ayuden a mejorar esos resultados.

\section{La calidad epistémica de los productos científicos}

¿Qué hace que una teoría, modelo o hipótesis sea buena desde el punto de vista científico? El enfoque de teoría de juegos sobre la construcción social del conocimiento proporciona dos intuiciones básicas para intentar responder a esta pregunta; no se trata de intuiciones muy profundas (en realidad, son casi triviales), pero también es cierto que son controvertidas al examinarlas desde algunos enfoques filosóficos o sociológicos, y por otro lado, también es verdad que existe una cierta tensión entre las dos intuiciones. La primera de ellas consiste en la afirmación de que, puesto que el análisis en términos de juegos asume que los científicos persiguen sus fines racionalmente, estamos obligados a asumir que los propios científicos tendrán una capacidad no despreciable de entender en qué consiste un "razonamiento correcto". Dicho de otro modo: si los científicos son tan inteligentes como para saber navegar el océano de sus relaciones sociales cuando luchan por conseguir recursos, publicaciones, honores, etc., entonces no deben ser necesariamente unos ineptos cuando tratan de descubrir, pongamos, las leyes que gobiernan un cierto fenómeno físico.

La segunda intuición, y más importante, es que la definición de la "calidad epistémica” de un producto científico no será fundamentalmente un problema para el filósofo de la ciencia (ni para el sociólogo o el economista de la ciencia), sino más bien para los propios científicos involucrados en la situación que 
estemos analizando, y también para el resto de los agentes relacionados con ella (industriales, políticos, ciudadanos, etc.). Por decirlo con una expresión latina: de epistemicibus gustibus non est disputandum. Tenemos que asumir que los científicos de carne y hueso tendrán algún tipo de "función de utilidad epistémica", probablemente no idéntica del todo la de cada uno de ellos, y la primera tarea de quien desee estudiar socialmente la producción del conocimiento científico no será tanto la de decir qué función de utilidad debería ser, sino más ben averiguar cuál es de hecho. La idea no es negar que pueda haber un montón de cosas interesantes que analizar desde un punto de vista filosófico sobre las posibles virtudes epistémicas de los ítems científicos: lo que nuestro enfoque nos sugiere es que no seamos, como filósofos, paternalistas sobre esta cuestión. Después de todo, los científicos son los mejores expertos que la sociedad tiene sobre la producción y el uso del conocimiento, y así, si alguien sabe en qué consiste el conocimiento y cómo distinguir ítems cognoscitivos "mejores" de otros "peores", esos son precisamente los científicos. Quizás ellos no son tan buenos a la hora de transformar este conocimiento tácito sobre la evaluación epistémica en conocimiento explícito (de hecho, cuando los científicos intentan explicar en qué consiste la "buena ciencia”, tienden a hacerlo peor que los filósofos), pero lo importante no es lo que revela lo que ellos dicen, sino lo que ellos hacen, su conducta como científicos. Tenemos que concentrarnos, pues, en lo que esta conducta revela sobre cuáles son sus criterios de una "buena práctica científica". Pero ¿cómo hacerlo? De hecho, yo diría que todas las teorías que han sido propuestas por los filósofos para intentar explicar la naturaleza del conocimiento científico, sus virtudes y su progreso, y a partir de ahí cuáles son las reglas metodológicas de la "buena ciencia", han sido refutadas al mostrar que, en la práctica real, los investigadores a menudo no se comportan como se supone que deberían hacer si estuvieran persiguiendo aquellas virtudes. Por ejemplo, los científicos no son habitualmente "falsacionistas" ni "confirmacionistas", pero tampoco "anarquistas" ni seguidores estrictos de la metodología Lakatosiana. Una buena parte de los debates del último medio siglo entre diversas escuelas de filosofía de la ciencia ha consistido en mostrar mediante ejemplos históricos o de la práctica científica actual, que los científicos no hacen lo que alguna teoría filosófica asume que deberían hacer. Mi sugerencia es que, en estos debates, casi todos los que intervienen tienen razón cuando están criticando las tesis de los rivales (más o menos como suceden en la política), pero están parcialmente equivocados cuando proponen sus propias explicaciones de la práctica científica. De este modo, el resultado de estos debates debemos considerarlo nuevamente como un abundante corpus de evidencia empírica sobre cómo se practica la ciencia, corpus en el que intentar encontrar algunas regularidades sobre o que los científicos consideran de hecho "buenas prácticas epistémicas".

El hecho de que los científicos se comportan de modos muy diferentes, y a menudo contradictorios, no es un argumento en contra de este fin: en cada sociedad puede haber prácticas y normas que se contradicen, tanto porque la gente tiene intereses, valores y preferencias distintas, como porque se enfrentan 
a situaciones y restricciones diferentes cada vez. En el caso de la ciencia, no es necesario descubrir muchas normas que todos los científicos en todas las épocas y lugares hayan considerado adecuadas; también sería interesante mostrar que, cuando se dan ciertas condiciones especificas, entonces tales y cuales normas de "buenas prácticas" tenderán a aceptarse. Nuestro objetivo principal como estudiosos de la ciencia debería ser, una vez que hemos organizado la experiencia de esta forma, intentar responder la siguiente pregunta: ¿qué función de utilidad hipotética explicaría del mejor modo posible la aceptación de precisamente estas ideas de "buenas prácticas" en cada situación, por parte de los científicos reales? Cada modelo económico hace unos cuantos supuestos razonables sobre las preferencias de los agentes, y los modelos de teoría de juegos de la ciencia no serán en esto diferentes de los demás. Mi conjetura (que no es nada original, y sí muy simplificada) es que la función de utilidad del científico "típico" contiene dos elementos principales: un componente "social" y otro "epistémica". El componente social puede contener diversas variables (ingresos, control sobre recursos, intereses de clase, valores políticos o humanitarios, etc.), pero el más importante de todos ellos creo que es el reconocimiento: los científicos se esfuerzan por ser reconocidos por sus colegas como buenos practicantes, o incluso "excelentes", de sus disciplinas; esto crea un incentivo para para ponerse de acuerdo, dentro de una comunidad científica, sobre cómo definir una "buena práctica", es decir, sobre cuáles son "las reglas del juego", pues, a falta de un acuerdo así, el "reconocimiento" se vuelve directamente imposible. El problema (para los científicos más que para los filósofos de la ciencia) es: ¿qué criterios utilizar para elegir esas reglas? Creo que las reglas más generales, básicas o universales, es decir, aquellas que nos permiten decir que un determinado tipo de práctica social cuenta como "ciencia" y no como otra cosa, deben de ser bastante similares en todas las comunidades científicas, aunque puedan tener bastantes diferencias en los detalles, y no deben de sufrir grandes cambios con el paso del tiempo; por esta razón, al decidir aceptar estas reglas, los científicos no pueden tener en cuenta muchos de sus intereses "sociales", pues es prácticamente imposible determinar de antemano si la adopción de ciertas reglas metodológicas (del más alto nivel) afectará de un modo o de otro la probabilidad de que un individuo determinado obtenga más o menos reconocimiento. Dicho de otro modo, las reglas más básicas de la ciencia tienden a ser aceptadas "tras el velo de la ignorancia", por usar la famosa metáfora rawlsiana. Por ese motivo tiendo a pensar que son los elementos epistémicos (y no los sociales) de la función de utilidad de los científicos los que tendrán más peso en la elección de ese tipo de normas. En otros escritos he propuesto una función de utilidad epistémica que podemos llamar "verosimilitud empírica" como una representación conjetural e idealizada de las preferencias epistémicas de los científicos, pero no es este el lugar de discutirla con detalle. 


\section{La eficiencia epistémica y las instituciones científicas}

Una cosa es definir en qué consiste la calidad científica de una teoría, modelo, etc., y otra muy distinta es determinar cómo de buena es esa teoría, etc., según aquella definición de calidad. Mi argumento de la sección anterior implica que tenemos que considerar a los científicos como nuestros mejores expertos sobre ambas cuestiones (aunque no nos digan a nosotros las respuestas: estas las tendremos que averiguar investigando su conducta), pero, incluso si ellos se ponen de acuerdo sobre qué hace que una teoría sea epistémicamente mejor que otra, ese acuerdo no garantiza por sí mismo que los resultados de la inversión que la sociedad hace en la ciencia vayan a tener necesariamente un nivel de calidad epistémica elevado. Esto dependerá de muchas otras variables, tales como el esfuerzo y el talento de cada científico, pero también de la eficiencia de las instituciones científicas. No me refiero aquí, en particular, a su eficiencia económica, sino a la eficiencia epistémica: ¿funcionan esas instituciones de tal manera que tienden a producir resultados de buena calidad? Para ilustrar de qué modo el enfoque de teoría de juegos puede ofrecer algunas respuestas a esta pregunta, examinaré brevemente los tres ejemplos que puse al final de la sección 2.

Estándares científicos: El proceso de elegir colectivamente un modelo, hipótesis, etc., como la solución correcta a un problema científico exige, como hemos visto, que la comunidad relevante se haya puesto de acuerdo sobre ciertos estándares que especifiquen el nivel mínimo de calidad epistémica que una "solución" debe tener para merecer serlo. ¿Elegirán los científicos un estándar "elevado", o más bien preferirán un estándar "bajo"? Por supuesto, esta pregunta sólo tiene sentido en comparación con algún criterio independiente de "altura", y el más obvio es el criterio contenido en las preferencias epistémicas de cada científico individual: supongamos que un científico que trabajase de manera aislada tuviera que decidir cuándo estará satisfecho con el número, la variedad y el rigor de las pruebas que una solución tiene que superar para ser aceptada por él como "la correcta"; este grado de calidad nos proporciona una especie de "marca" con la que comparar el resultado del estándar que ese mismo científico elegiría colectivamente en coordinación con el resto de miembros de su comunidad. Los modelos que he presentado sobre este tema muestran que, en el caso de que la investigación se organice institucionalmente como una especie de competición o "carrera por el reconocimiento", es razonable esperar que el estándar epistémico elegido colectivamente tenderá a ser más exigente que los estándares que cada científico preferirían de modo individual. Es decir, la búsqueda de "soluciones" en ambientes competitivos termina proporcionando a la sociedad soluciones que son epistémicamente mejores que las que se propondrían en un ambiente no competitivo. De hecho, quizás las soluciones sean a veces "demasiado" buenas, en el sentido de que nosotros, los ciudadanos, quizá preferiríamos que los científicos se esforzasen un poco menos por mejorar la calidad de esas soluciones, a cambio de que encontrasen soluciones (un poco peores) a más problemas. De todas formas, la consecuencia principal que podemos sacar de este análisis es que, desde un 
punto de vista epistemológico, no hay ninguna razón para afirmar que la búsqueda competitiva de reconocimiento lleva a los científicos a aceptar, por término medio, teorías que no son "lo suficientemente buenas" como conocimiento.

Elección de teoría: Como vimos en la sección 3, si la aceptación de una tesis por parte de un científico depende de cuáles de sus colegas también la aceptan, puede darse el caso de que más de un resultado sea "socialmente" posible. Por ejemplo, puede ocurrir que el que se acepte la hipótesis $\mathrm{H}$ por un $20 \%$ de los miembros de la comunidad sea un equilibrio (cada miembro está contento con su decisión, dadas las decisiones de los demás), pero también que lo sea el que la hipótesis sea aceptada por el $70 \%$ de los miembros (y que cualquier otro porcentaje no sea un equilibrio posible); en un caso así, cuál de los dos equilibrios tengan lugar de hecho, dependerá de causas históricas (o sea, de cuáles eran las situaciones de partida). Esto parece constituir una razón a favor de cierto grado de relativismo: el consenso científico es el que es, pero, con la misma información y con las mismas relaciones sociales, podría haber sido otro. De todas formas, es un relativismo bastante limitado, puesto que hay muchos más estados imaginables que no son un equilibrio que estados que sí que lo son. La situación es aún peor: también puede ocurrir que todos los miembros de la comunidad estén de acuerdo en que la hipótesis H' sea mejor que la hipótesis $\mathrm{H}$ (no hablo de hipótesis en competición, sino que cada una de ellas sea respuesta a un problema distinto), y en cambio $\mathrm{H}$ sea aceptada por muchos más miembros que H’. Digamos que en un caso así habría ocurrido una ineficiencia epistémica. Las buenas noticias son que también es posible probar que, a medida que una hipótesis ha haciéndose mejor (p.ej., porque se acumulan más pruebas a su favor), no sólo los grados de aceptación de equilibrio correspondientes a esa hipótesis se hacen mayores, sino que al final tiende a quedar solo uno, y muy elevado. Así pues, es posible que haya cierto grado de "relatividad" en el estado del conocimiento científico en un momento dado (al fin y al cabo, lo que llamamos "conocimiento" es en el fondo conjetural), pero el incremento de la información empírica puede ir haciendo disminuir esa relatividad.

La construcción de un hecho empirico: Supongamos que has llevado a cabo un experimento, y estás planificando cómo reflejarlo en un artículo. Hoy en día es una obviedad, en el campo de los science studies, decir que en un caso así, tendrás varias maneras distintas de hacerlo, pues los hechos "no hablan por sí mismos", sino que deben ser "interpretados". Por ejemplo, puedes reflejar tu trabajo como un descubrimiento muy importante, que fuerza a tu comunidad a buscar una nueva explicación para él y a revisar otras teorías, o puedes presentarlo como algo más o menos trivial. El problema es que, cuanto más "radical" sea la interpretación que elijas, menos "creíble" será, o sea, "peor confirmada" estará por los datos empíricos que puedas presentar a tus colegas. Como vimos más arriba, la mera existencia de más de una alternativa no implica que "todas valgan lo mismo": algunas de ellas serán "mejores" que otras desde el punto de vista epistémico de unos u otros científicos, mientras que otras serán "mejores" según las ventajas 
"sociales" que puedan proporcionar a cada uno. Esta pluralidad de evaluaciones no debe confundirse con algún tipo de "indiferencia radical": los modelos de teoría de juegos analizan precisamente aquellas situaciones en las que los individuos tienen intereses diferentes, y nos ayuda a analizar qué resultado colectivo se obtiene en esos casos; y comparando el resultado así determinado con el valor que otros resultados posibles habrían tenido para los propios individuos, el modelo también nos permite evaluar la eficiencia de esa interacción. Es decir, un resultado puede ser ineficiente en el sentido de que los propios científicos reconocerían que habrían preferido otro, sólo que, tal como funcionan sus instituciones, la interacción en la que han intervenido ha llevado al resultado primero. En el caso que analizo en los modelos a los que se refiere esta sección, lo que puede probarse es que se da un incentivo para que el autor de un artículo acabe decidiendo elegir una interpretación de sus resultados que es peor, desde el punto de vista epistémico de los lectores del artículo, que algunas otras interpretaciones que podía haber elegido. Es de esperar, por tanto, que los científicos tiendan a desarrollar instituciones que favorezcan el punto de vista de los lectores, o "usuarios", de los artículos, más que los de sus autores (no olvidemos que cada científico es más a menudo "usuario" de los artículos producidos por otros, que autor de artículos que otros colegas “usarán”).

\section{Conclusión}

¿Es la ciencia "racional”? ¿Es el conocimiento científico "objetivo”? El enfoque de teoría de juegos sugiere que estas preguntas deberían ser reformuladas del modo siguiente: ¿Están los métodos e instituciones científicas diseñados -o han evolucionado- de manera "eficiente"? Y, ¿̨te parece a ti que los fines epistémicos de los científicos son adecuados? Si tu respuesta a la última pregunta es "si", entonces una respuesta afirmativa a la primera cuestión debería ser suficiente para satisfacer (a un nivel razonable) nuestras posibles dudas sobre la racionalidad y la objetividad de la ciencia. Si la cuestión a la primera pregunta fuese negativa, entonces podríamos emplear el enfoque de teoría de juegos para intentar hallar dónde están fallando exactamente las instituciones científicas, y cómo podrían ser mejoradas. Por otro lado, si tu respuesta a la última pregunta es "no", o sea, si tú crees que los científicos deberían tener otros valores epistémicos distintos a los que de hecho tienen, entonces lo que tú debes en el argumento sobre la racionalidad de la ciencia es una explicación de cuáles crees que esos valores epistémicos deberían ser, y un conjunto de modelos de instituciones científicas que muestre cómo debería estar la ciencia organizada de manera que fuese eficiente en la consecución de esos valores epistémicos. 


\section{Agradecimientos}

Este artículo se ha beneficiado de los proyectos de investigación PRX1400007 y FFI2014-57258-P.

\section{Referencias bibliográficas}

Fernández Pinto, M. (2016). Economics Imperialism in Social Epistemology: A Critical Assessment. Philosophy of the Social Sciences, 46, 443-472.

Ferreira, J. L., and J P. Zamora Bonilla (2006). An Economic Theory of Scientific Rules. Economics and Philosophy, 22, 191-212.

Zamora Bonilla, J. P. (1999). The Elementary Economics of Scientific Consensus. Theoria, 14, 461-88.

Zamora Bonilla, J. P. (2002a). Scientific Inference and the Pursuit of Fame: A Contractarian Approach. Philosophy of Science, 69, 300-23.

Zamora Bonilla, J. P. (2006a). Science as a Persuasion Game. Episteme, 2, 189201.

Zamora Bonilla, J. P. (2006b). Rhetoric, Induction, and the Free Speech Dilemma. Philosophy of Science, 73, 175-93.

Zamora Bonilla, J. P. (2007). Science Studies and the Theory of Games. Perspectives on Science, 14, 639-71.

Zamora Bonilla, J. P. (2011). The Economics of Scientific Knowledge. En U. Mäki (Ed.), Philosophy of Economics. Handbook of the Philosophy of Science, vol. 13 (pp. 759-798). Ámsterdam: Elsevier. 
